\title{
New Agents to Treat Chronic Lymphocytic Leukemia
}

TO THE EDITOR: Roberts et al. (Jan. 28 issue ${ }^{1}$ describe improved outcomes for patients with relapsed chronic lymphocytic leukemia (CLL) who were treated with venetoclax, a drug that targets BCL2 protein. Patients with CLL are known to have skeletal problems. Data for 21,015 patients recently presented at the 57th Annual Meeting of the American Society of Hematology convincingly showed that even untreated patients with CLL have poor bone health and a significantly increased risk of fracture, particularly of the spine and pelvis. ${ }^{2}$ Suppression of BCL2 has been shown to accelerate osteoblast differentiation and improve bone health when tested in preclinical models. ${ }^{3}$ On the basis of these observations, it seems that suppressing BCL2 with venetoclax has the potential to improve bone strength in patients with CLL, a hypothesis that should encourage the extraction of information on bone health from ongoing studies. When new clinical trials of venetoclax are designed, we propose that serum markers of bone metabolism be included in the protocol.

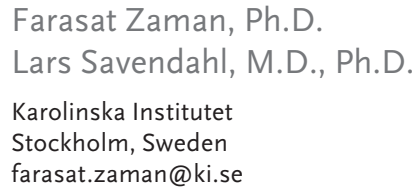

No potential conflict of interest relevant to this letter was reported.

1. Roberts AW, Davids MS, Pagel JM, et al. Targeting BCL2 with venetoclax in relapsed chronic lymphocytic leukemia. $\mathrm{N}$ Engl J Med 2016;374:311-22.

2. Olszewksi AJ, Gutman R, Eaton CB. Increased fracture risk in patients with untreated chronic lymphocytic leukemia (CLL): a population-based analysis. Presented at the 57th Annual Meeting of the American Society of Hematology (ASH), Orlando, FL, December 4-8, 2015. abstract.

3. Moriishi T, Kawai Y, Komori H, et al. Bcl2 deficiency activates FoxO through Akt inactivation and accelerates osteoblast differentiation. PLoS ONE 2014;9(1):e86629.

DOI: 10.1056/NEJMc1602674

TO THE EDITOR: Byrd et al. (Jan. 28 issue) ${ }^{1}$ describe the promising safety and efficacy of acalabrutinib, a selective inhibitor of Bruton's tyrosine kinase (BTK) in patients with relapsed CLL. Ibrutinib, the first-in-class irreversible BTK inhibitor, has transformed the clinical management of CLL. ${ }^{2}$
However, the kinome of ibrutinib is broad, ${ }^{3}$ which may result in substantial toxic effects. ${ }^{2} \mathrm{Se}-$ lective BTK inhibitors include ONO/GS-4059 and acalabrutinib. ${ }^{4}$ We recently reported a phase 1 trial with ONO/GS-4059 and, in parallel with the acalabrutinib study, selective BTK inhibition was associated with a marked reduction in toxic effects. ${ }^{4}$ In terms of efficacy, a maximal tolerated dose among patients with CLL was not established in either study; nearly all the patients with CLL entered durable remissions with either molecule. Updated survival data for the 26 patients treated with ONO/GS-4059 who could be evaluated are provided in Figure 1; the median length of treatment with ONO/GS-4059 at the time of this analysis was 29.9 months. Whether long-term efficacy in CLL relates to maintained, full BTK occupancy remains unknown, but inhibition of kinases other than BTK does not appear to be necessary. To date, Richter's transformation has not developed in any patients receiving $\mathrm{ONO} /$ GS-4059 for more than 1 month. Selective BTK inhibition may have advantages over ibrutinib not only in reduced toxic effects but also in the development of new combination therapies.

Harriet S. Walter, M.D.

Ernest and Helen Scott Haematological Research Institute Leicester, United Kingdom

Gilles A. Salles, M.D., Ph.D.

Hospices Civils de Lyon

Lyon, France

Martin J.S. Dyer, D.Phil.

Ernest and Helen Scott Haematological Research Institute Leicester, United Kingdom

mjsd1@le.ac.uk

Dr. Salles reports receiving lecture fees and payment for serving on an advisory board from Gilead and research funding from ONO; and Dr. Dyer, receiving lecture and travel fees from Gilead and research funding from ONO. No other potential conflict of interest relevant to this letter was reported.

1. Byrd JC, Harrington B, O'Brien S, et al. Acalabrutinib (ACP196) in relapsed chronic lymphocytic leukemia. N Engl J Med 2016;374:323-32.

2. Wiestner A. The role of B-cell receptor inhibitors in the treatment of patients with chronic lymphocytic leukemia. Haematologica 2015;100:1495-507.

3. Honigberg LA, Smith AM, Sirisawad M, et al. The Bruton tyrosine kinase inhibitor PCI-32765 blocks B-cell activation and is efficacious in models of autoimmune disease and B-cell malignancy. Proc Natl Acad Sci U S A 2010;107:13075-80. 


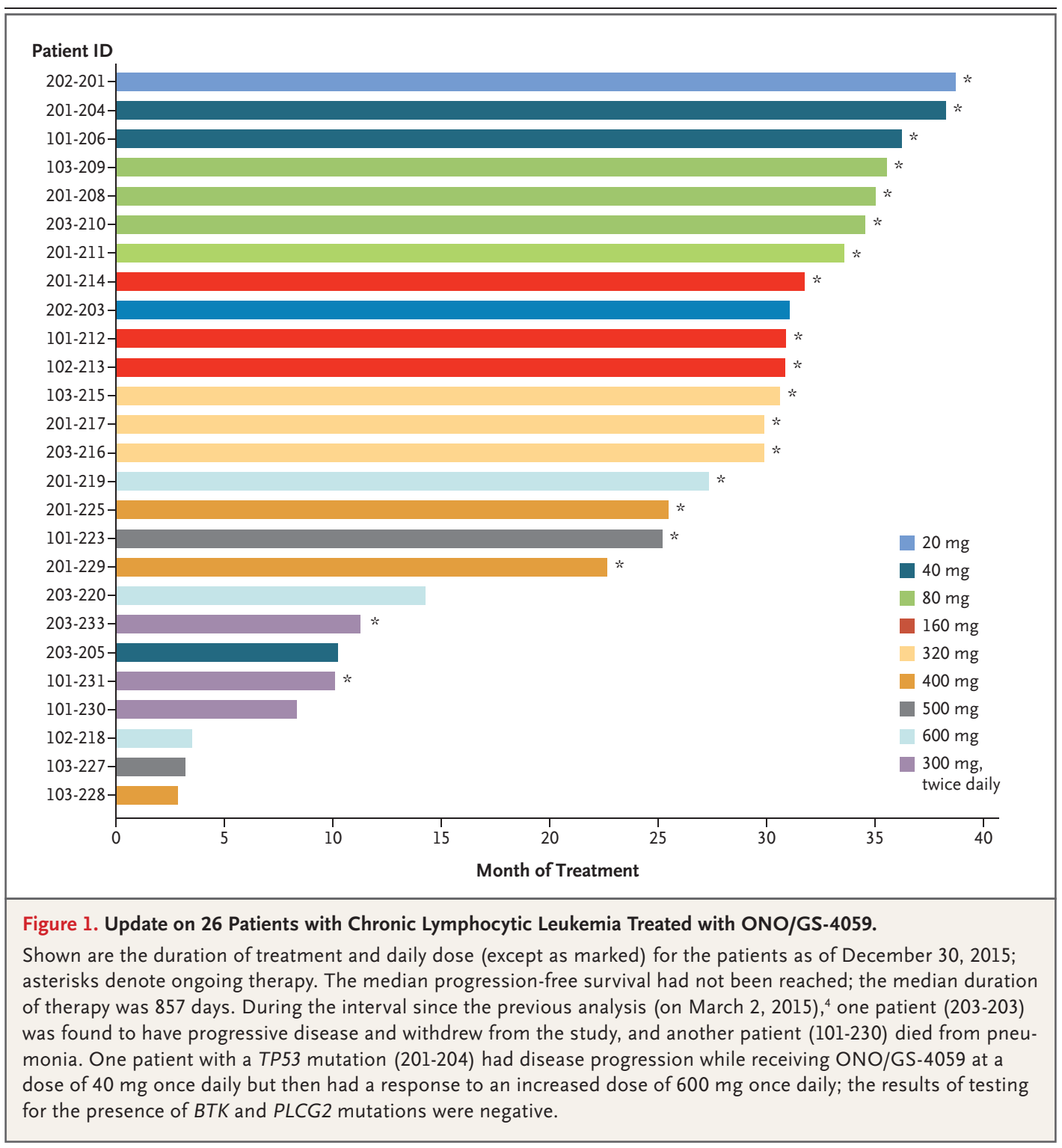

4. Walter HS, Rule SA, Dyer MJ, et al. A phase 1 clinical trial of the selective BTK inhibitor ONO/GS-4059 in relapsed and refractory mature B-cell malignancies. Blood 2016;127:411-9.

DOI: 10.1056/NEJMc1602674

DR. ROBERTS AND COLLEAGUES REPLY: In response to Zaman and Savendahl: in the venetoclax trial, fractures were reported in 6 of 116 patients (two femoral, two upper limb, one lumbar vertebral, and one facial). The trial design and relatively short-term median follow-up of 17 months preclude any precise estimation of the rate of bone fracture for patients receiving venetoclax therapy. The clinical implications of the genetic data are unclear, given the multiplicity of effects of loss of BCL2 on both osteoblasts and osteoclasts. ${ }^{1,2}$ Fur- thermore, unlike genetic loss, pharmacologic antagonism may not deplete BCL2 function completely. Thus, we agree that it could be informative to include biomarkers of bone turnover in future studies of BCL2-inhibitor therapy, and estimates of fracture incidence could be generated from pooled safety data from current venetoclax trials after longer follow-up.

Andrew W. Roberts, M.B., B.S., Ph.D.

Royal Melbourne Hospital

Parkville, VIC, Australia

andrew.roberts@mh.org.au

Matthew S. Davids, M.D.

Dana-Farber Cancer Institute

Boston, MA 